\title{
2D and 3D Analyses of Metal Oxide Thin Films Examined by Atomic Force Microscope
}

\author{
FAEZAHANA Mokhter ${ }^{1 a}$, NAFARIZAL Nayan ${ }^{1}$, \\ JIA WEI Low ${ }^{1}$, NORHIDAYAH Che Ani ${ }^{1}$, MOHD ZAINIZAN Sahdan ${ }^{1}$, \\ MOHD KHAIRUL Ahmad ${ }^{1}$, ALI YEON Md Shakaff ${ }^{2}$, AMMAR Zakaria², \\ and AHMAD FAIZAL Mohd Zain ${ }^{3}$ \\ ${ }^{1}$ Microelectronic and Nanotechnology - Shamsuddin Research Centre (MiNT-SRC), \\ Universiti Tun Hussein Onn Malaysia, 86400 Parit Raja, Batu Pahat, Johor, Malaysia \\ ${ }^{2}$ Centre of Excellence for Advanced Sensor Technology, Universiti Malaysia Perlis, \\ 02600 Arau Perlis, Malaysia \\ ${ }^{3}$ Faculty of Manufacturing Engineering, Universiti Malaysia Pahang, \\ 26300 Gambang Kuantan, Pahang Darul Makmur, Malaysia \\ afaezahana@uthm.edu.my
}

Keywords: metal oxide thin film, atomic force microscope, magnetron sputtering deposition

\begin{abstract}
Atomic force microscope (AFM) is a useful tool to capture the two- and three-dimensional image of height and size of nanostructured thin film. It operate by measuring the forces between a sharp tip and surface of the measured sample. In addition, AFM is equipped with powerful software for image processing to interpret experimental results in detail. For example, by using the height and scanning length parameters of measured sample, average roughness and root mean square roughness can be evaluated. In the present works, the effect of image flattening process toward the surface roughness and surface fluctuations of metal oxide thin films will be presented. Set of samples were prepared by magnetron sputtering deposition and sol-gel coating techniques. In gas sensor industries using metal oxide thin film, surface roughness of metal oxide thin films are very important in order to improve the sensitivity and respond time of gas sensor. Therefore, optimization of thin film deposition and characterization are very important. The correlation between the three-dimensional image and thin film deposition and image processing parameters will also be presented.
\end{abstract}

\section{Introduction}

Metal oxide semiconductors were of interest to scientist for decades. Based to some earlier reports of gas sensing design, almost any of the metal oxide could be used for solid-state gas sensor. Theoretically there are no limitation for using any metal oxide for solid-state gas sensor independently of their physical, chemical, structural or electrical properties[1]. For this purpose, we need to understand and control the interaction of surface properties of prepared metal oxide.

Gas sensors are widely used in many important area such as environmental monitoring, automotive applications and air conditioning. Air pollution issue has been one of the world political concern since the rapid development of China and India. In recent years, research effort has been directed toward the development of nano-dimensional gas-sensing devices for practical applications in toxic gas and air pollutant monitoring. Researchers have been focusing for gas sensor with higher sensitivity[2]. In addition, detection of air pollutant at relatively low cost is becoming very important.

The main focus of this paper is on the physical properties of metal oxide which can be used for gas sensing applications. Different deposition techniques, which is sputtering and sol-gel have been used to prepare these thin films. In nanotechnology research area, atomic force microscope (AFM) has become a valuable system to measure the surface properties such as roughness and electrical 
properties of materials[3]. AFM is a powerful tool to capture the two- and three-dimensional images of height and size of nano-structured thin film. It operate like a profilometer by measuring the forces between a sharp probe and surface at a very short distance between probe and measured sample. Using a very high resolution height and scanning length parameters of measured sample, average roughness and root mean square roughness were calculated. In general, AFM is equipped with powerful software for image analyzing to interpret the experimental result in details.

\section{Experimental details}

Copper oxide thin film was deposited on silicon wafer substrate using reactive magnetron sputtering plasma. The magnetron sputtering source was made of cylindrical permanent magnets attached to an indirect water cooling system. The distance of substrate surface was $13 \mathrm{~cm}$ from the target. Prior to deposition, silicon wafer was oxidized using wet oxidation process to form $1 \mu \mathrm{m}$ thick $\mathrm{SiO}_{2}$ layer as the insulator. The sputter target size is a 3 inches diameter of pure $(99.99 \%$ purity) copper target. By using a pure copper target, one can easily control the stoichiometric of deposited thin film using the oxygen-to-argon ratio. The sputter chamber was evacuated to base pressure of $5 \times 10^{-6}$ Torr using vacuum turbo pump and backed by rotary pump. The required sputtering gas (argon, Ar) and reactive gas (oxygen, $\mathrm{O}_{2}$ ) were admitted into the chamber by using the mass flow controller which is attached to the top of the vacuum chamber. During the deposition, Ar flow rate and total chamber pressure were fixed at $50 \mathrm{sccm}$ and $22.5 \mathrm{mTorr}$, respectively. Sputtering plasma was produced by a $13.6 \mathrm{MHz}$ rf magnetron discharges with an automated matching network. The rf discharge power was fixed at $400 \mathrm{~W}$. For a comparison, zinc oxide thin film was prepared using sol-gel deposition. The preparation technique are reported elsewhere [4].

Surface roughness of deposited thin films were evaluated using AFM. Generally, the non-contact atomic force microscope (NC-AFM) mode was selected to prevent contamination on AFM tip. The height on the images $(Z)$ recorded in NC-AFM will represent the topography of sample surface. Force interaction between the NC-AFM tip and the sample surface must be optimized for accurate topography of samples and prevent tip damage. The AFM used in this study was from Park System, Korea. The sample was mounted carefully on sample holder using a double-sided tape. Generally, this procedure of mounting causes a tilted result of structure surface. In order to eliminate such deficiency of structure, images were processed using flattening second-order processing software proposed with AFM system. Note that the more manipulation of image processing will result in less accuracy of surface information. Therefore, an optimum order of processing has to be selected.

\section{Results and discussion}

Figure 1 shows 2-D and 3-D images of copper oxide thin film deposited by magnetron sputtering. The film thickness was approximately $150 \mathrm{~nm}$. The image was just as captures during a real-time operation (raw image). The AFM images reveal that copper oxide deposited using magnetron sputtering have a uniform structure. It have sharp peaks at nano scale size as clearly seen by 3 -D image in figure 1 . From the AFM image, thin film roughness is determined from $R_{q}=$ $\sqrt[2]{\sum Z-Z_{\text {ave }} / N}$, where $R_{q}$ is the standard deviation of $Z$ values, $Z_{\text {ave }}$ is the average of $Z$ values, $Z$ is the current height and $N$ is the number of points within the given area. Although no significant different was observed from the raw and flattened images of figure 1 and 2 , the value of $R_{q}$ for figure 1 and 2 were 4.47 and $4.39 \mathrm{~nm}$, respectively. The surface roughness evaluated from raw image and flattened image was slightly different. Therefore, careful handling during the sample testing preparation is very important. 

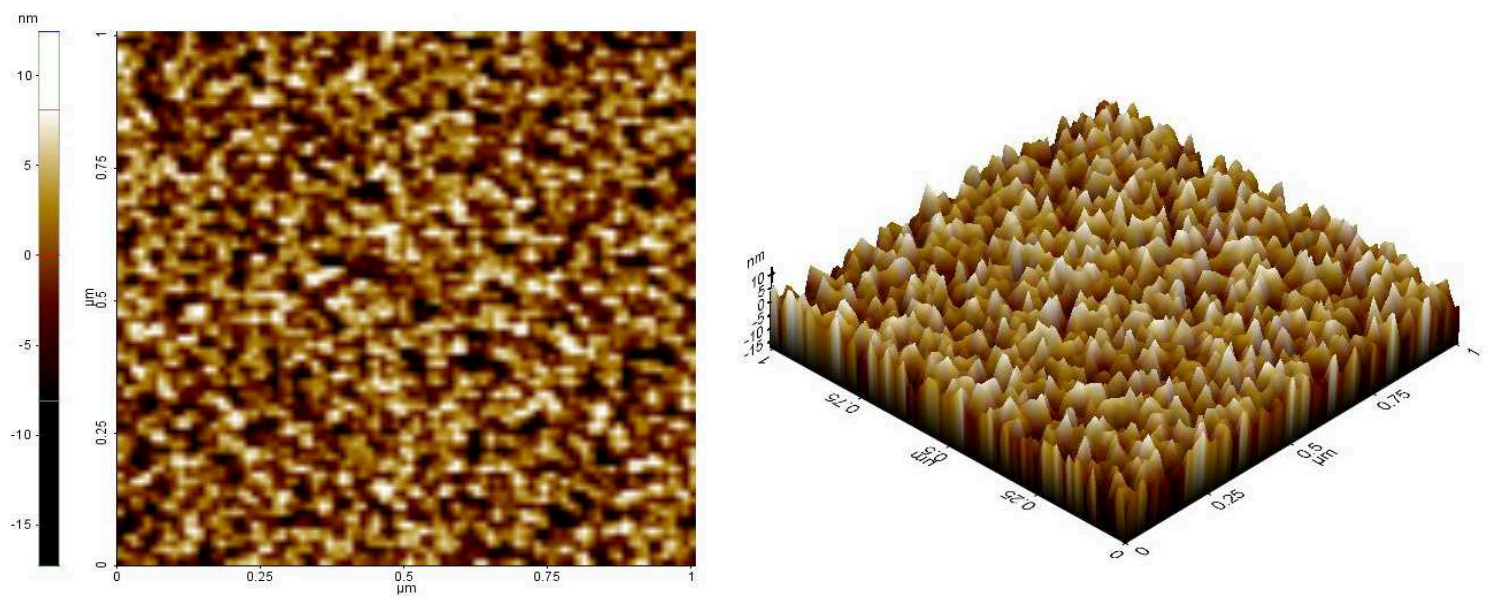

Figure 1.2D and 3D image of raw image from copper oxide thin film deposited by magnetron sputtering.
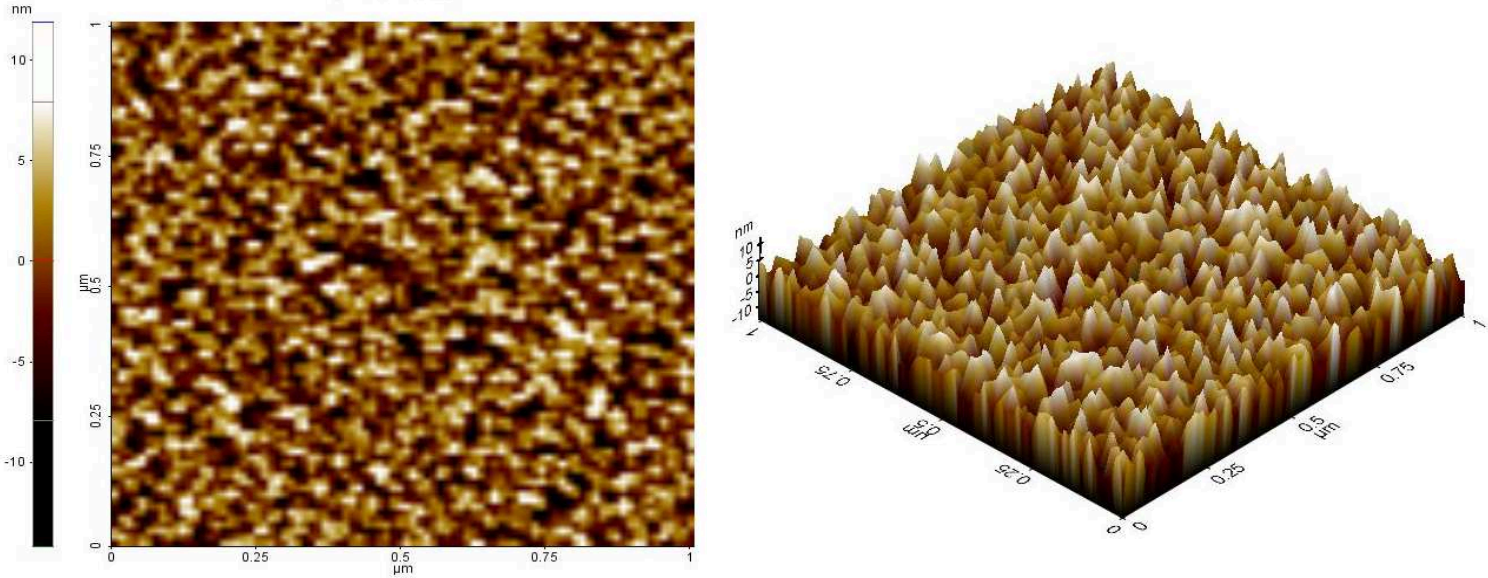

Figure 2. 2D and 3D image of flattened copper oxide thin film deposited by magnetron sputtering.

Figure 3 shows the 2-D and 3-D images of flattened image for zinc oxide sample prepared using sol-gel technique. The grain size of each particles were larger than the sample prepared using magnetron sputtering technique. This is common due to the nature of deposition in vacuum and in atmospheric pressure conditions. In addition the thin film prepared using sol-gel technique was not uniform. The value of $R_{q}$ for figure 3 was $3.45 \mathrm{~nm}$. To give a clear view of grain size, one can plot the line profile of AFM images as shown in figure 4.
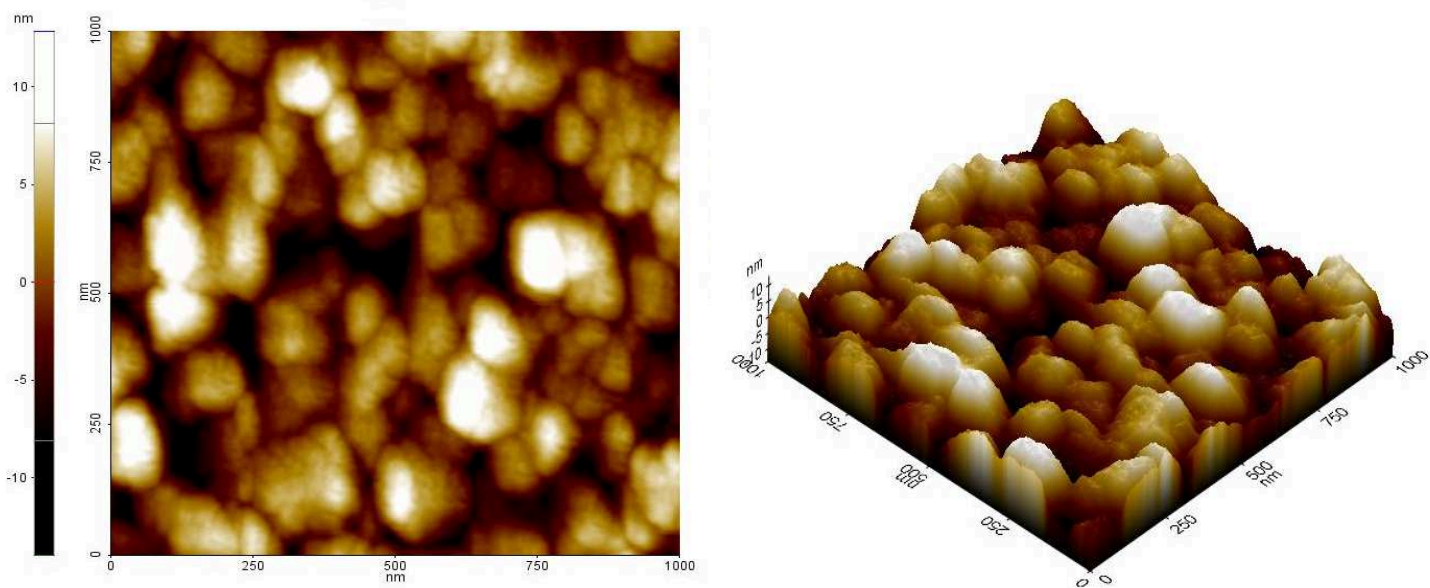

Figure 3. 2D and 3D image of flattened zinc oxide thin film deposited by sol-gel technique. 

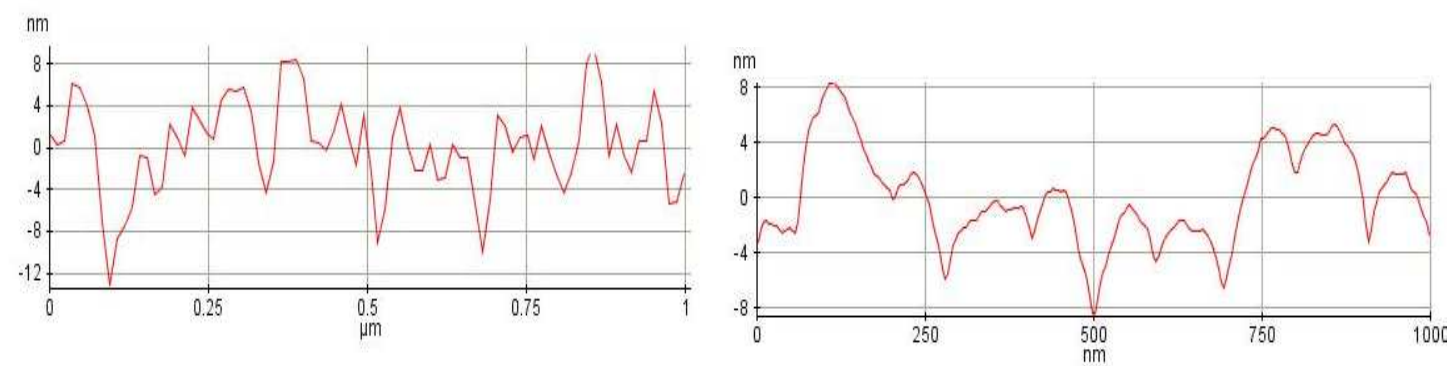

Figure 4. Line profile of flattened images of metal oxide thin films deposited by magnetron sputtering (left) and sol-gel (right) technique.

\section{Conclusion}

Metal oxide thin films have been successfully deposited using magnetron sputtering plasma. Surface topography and thin film roughness were evaluated using AFM analysis. Image processing plays an important in AFM analysis and have to be optimized in order to prevent mistake during data interpretation. Surface roughness of thin film prepared by magnetron sputtering and sol-gel deposition had a large different.

\section{Acknowledgment}

The present work was supported by Malaysian Technical Universities Network: Centre of Excellence Grant and Fundamental Research Grant Scheme of Ministry of Education Malaysia.

\section{References}

[1] G. Korotcenkov, "Metal oxides for solid-state gas sensors: What determines our choice?," Mater. Sci. Eng. B, vol. 139, no. 1, pp. 1-23, Apr. 2007.

[2] H. Xu, X. Liu, D. Cui, M. Li, and M. Jiang, "A novel method for improving the performance of ZnO gas sensors," Sensors Actuators B Chem., vol. 114, no. 1, pp. 301-307, Mar. 2006.

[3] G. Kavei, Y. Zare, and a Mohammadi Gheidari, "Evaluation of surface roughness and nanostructure of indium tin oxide (ITO) films by atomic force microscopy.," Scanning, vol. 30, no. 3, pp. 232-9, 2008.

[4] S. H. Kamaruddin, M. Z. Sahdan, K. Y. Chan, S. N. M. Tawil, N. Nayan, "Surface morphology and optical properties of $\mathrm{ZnO}$ tilms synthesis using different solvent," Advanced Material Research, vol. 832 pp. 478-482, 2014. 